\title{
OPINION
}

\section{Art: a forum to display scientific concepts}

\author{
D.T.U. Abeytunga ${ }^{{ }^{*}}$ and Donna K. Friel ${ }^{2}$ \\ ${ }^{1}$ Department of Chemistry, University of Colombo, Colombo -03. \\ 2 Department of Chemistry, Boston College, Massachusetts, U.S.A.
}

Do you have enough empty wall space in the corridors of buildings at your universities and schools? I am sure the answer to this question is a "yes". Can these empty walls be used to stimulate the young minds through colourful pieces of art? People in general spontaneously try to explain things they experience. An art, for example, can provoke many ideas and thoughts and may be a powerful way to increase curiosity towards a specific subject. Can art be used as a forum to articulate ideas, theories, and experimental observations related to general science and chemistry in particular? This would be a rather different approach to the existing models for teaching such as Problem Based Learning. Art can never replace the existing teaching methods but would provide a parallel method that would capture the minds of students and inculcate the habit of observation and discussion while passing the artwork in the corridor. Concept building through art should not culminate at the hallway, but rather can act as a framework with which new theories and ideas are elaborated upon in the classroom.

If students have been intrigued or motivated by an artwork, this could lead to a focused study in a particular area. As scientists, let us investigate whether such nonverbal display on the walls of learning facilities could assist in cognitive learning strategies.

\section{Can we explain chemistry jargon using art ?}

Let us look at the painting given in Figure 1, the flying butterflies, by the author of this article. Are these two wall hangings identical? If not what is the relationship between the two paintings? The two wall hangings are mirror images of one another.

In general, in life and science, objects occupy space; specifically speaking organic molecules occupy space.
A very simple organic molecule having one carbon with four different groups can be arranged in two different orientations as shown in Figure 2. It is important to note, that these four different groups maintain a tetrahedral geometry.

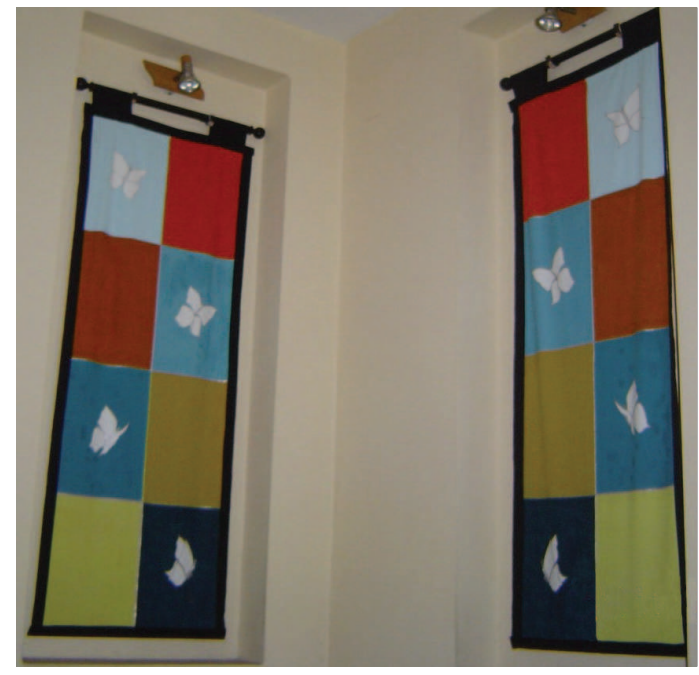

Figure 1: The flying butterflies
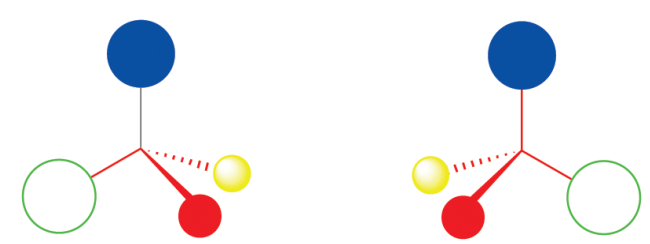

Figure 2: A carbon with four different groups could arrange in space in two different ways 
Can we superimpose the two molecules in Figure 2? No, they are non-super imposable mirror images. This relationship is called enantiomerism. The individual molecules in Figure 2 are called enantiomers. This word is derived from the Greek word "enantio", meaning opposite.

This relationship of enantiomerism is of utmost importance in all living systems as one enantiomer can behave quite differently than the other. For example, the amino acid (+)-leucine (one enantiomer) has a sweet taste where as (-)-leucine (the other enantiomer) has a bitter taste.

Would the above painting be of any use, if it is hung outside a lecture hall where organic chemistry is taught to undergraduate students? Can we use similar paintings to assist students to comprehend other concepts in science?

\section{Art O'Spectroscopy}

Spectroscopy is a wonderful technique invented by scientists to unravel molecular architecture of simple and complex molecules. A very popular technique used in structure elucidation, is called Nuclear Magnetic Resonance Spectroscopy (NMR Spectroscopy). A common technique of NMR Spectroscopy is called ${ }^{1} \mathrm{H}-{ }^{1} \mathrm{H}$ COSY, a 2-D NMR technique which allows one to extract information regarding proton connectivity based on spin-spin coupling of neighboring protons. Figure 3 depicts a COSY spectrum of a simple organic molecule in the form of a piece of art (designed and painted by the author in 2005). Those students who have studied

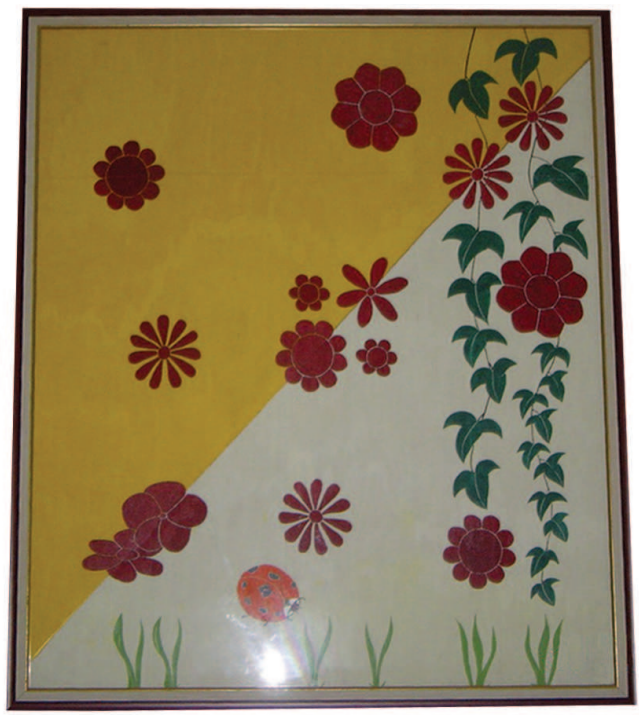

Figure 3: The flower garden spectroscopy can solve this structure of the organic molecule using the explanation given below. Again what if we post this piece of artwork in an environment where ideas of molecular structure and NMR Spectroscopy are discussed. Would this puzzle provide positive attractive stimulation for those who are familiar with spectroscopic techniques?

The diagonal and the off diagonal peaks are given as flowers. The number of leaves in the left most wine gives the number of protons in the molecule. The number of leaves in the next gives the number of carbons in the molecule. In spectroscopic terms these wines could be considered as T1 noise. There is one oxygen in the unknown and it is depicted as a bug in the garden. In a COSY spectrum this may be an artifact. The $\mathrm{v}_{2}$ axis is given at the bottom. The number of protons under each peak can be obtained by counting the number of leaves in each plant.

The solution to the COSY exercise is 3-heptanone.

The examples stated above are in correlation with organic chemistry; however, art is not limited to a specific area.

\section{Can a painting be used to express the idea of binary numbers?}

Binary numbers is a concept in mathematics, which can easily be illustrated using an art work. Binary numbers can be used to represent any number by using only 2 digits: 0 and 1 . Each digit " 1 " in a binary number represents a power of two and each " 0 " represents zero. Here is a very simple description of binary arithmetic.

$$
\begin{aligned}
& 1111=2^{3}+2^{2}+2^{1}+2^{0}=8+4+2+1=15 \\
& 0101=0+2^{2}+0+2^{0}=0+4+0+1=5 \\
& 1010=2^{3}+0+2^{1}+0=8+0+2+0=10
\end{aligned}
$$

To represent bigger whole numbers (integers) more places (bits) in the binary number (e.g. $10000001=129$ ) are needed. This concept is used every day in storing information in computer hardware. How can we express the concept of binary numbers on a painting? The two digits " 1 " and " 0 " can be easily depicted by using two different colors. Let us say " 1 " is black and " 0 " is white. This conceptual idea is demonstrated in the following fabric painting "The blossoming child" designed and painted by author in 2004 (Figure 4).

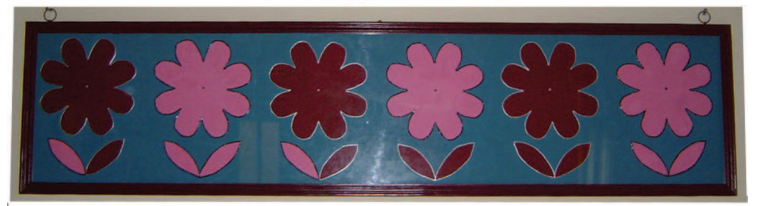

Figure 4: The blossoming child 
The flowers in the painting are the child and the two leaves depict the parents. In the color scheme, red indicates the digit " 1 " and the pink indicates the digit " 0 ". This painting enables the viewer to use binary number system in an unconventional way (from left to right) to calculate the day of the birth of the child and the parents. The flower head represents the child's birthday, the right leaf represents the mother's birthday and the left leaf represents the fathers birthday.

$$
\begin{aligned}
\text { Child }= & 101010=2^{0}+0+2^{2}+0+2^{4}+0=1+0+4 \\
& +0+16+0=21
\end{aligned}
$$

Father $=000110=0+0+0+2^{3}+2^{4}+0=0+0+0$ $+8+16+0=24$

Mother $=101110=2^{0}+0+2^{2}+2^{3}+2^{4}+0=1+0+4$ $+8+16+0=29$

In summary, art as a learning tool in science, specifically in chemistry can help motivate and stimulate positive and productive thinking. Also, it provides an opportunity for open discussions in relation to art and science in general in the hopes of providing students and teachers with the stimulation and desire to learn. 